\title{
Reply to Letter to Editor
}

\section{Orthopaedic Surgeons Prefer to Participate in Expertise-based Randomized Trials}

\author{
Dianne Bryant MSc, PhD, \\ P. J. Devereaux MD, PhD, FRCPC, \\ Elzbieta Bednarska BHSc (Hon)
}

Published online: 22 October 2008

(C) The Association of Bone and Joint Surgeons 2008

We thank Dr. Biau and Professor Porcher for their comments regarding our recently published article, "Orthopaedic Surgeons Prefer to Participate in Expertisebased Randomized Trials" [1]. We wish to first emphasize we do not view the expertise-based design as a panacea for all challenges related to surgical trials. We do, however, view the expertise-based design as an option that holds substantial promise, under circumstances outlined in our paper, to provide less biased results than the conventional design and to enhance feasibility of conducting randomized controlled trials (RCTs) in surgery.

Drs. Biau and Porcher acknowledge differential expertise bias will affect the results of a conventional RCT, but they feel assured statistics can resolve the problem. To avoid confounding and assure optimal statistical adjustment, however, researchers first must establish all of the predictors of outcome to determine what effect is attributed to the surgeons, and then each surgeon must provide a sufficient number of cases in a large multicenter study to determine whether an individual surgeon was contributing an effect.

D. Bryant $(\bowtie)$

School of Physical Therapy, Faculty of Health Sciences \& Division of Orthopaedic Surgery, Faculty of Medicine and Dentistry, University of Western Ontario, London, ON, Canada e-mail: dianne.bryant@uwo.ca

D. Bryant, P. J. Devereaux

Department of Clinical Epidemiology \& Biostatistics, Faculty of Health Sciences, McMaster University, Hamilton, ON, Canada

P. J. Devereaux

Department of Medicine, Faculty of Health Sciences, McMaster University, Hamilton, ON, Canada

E. Bednarska

Faculty of Health Sciences, McMaster University, Hamilton,

ON, Canada
Given that these circumstances are uncommon, we are less confident statistics can resolve the problem. Regarding the simulation described by Drs. Biau and Porcher, it is difficult to know exactly what it shows given the limited information provided. Further, it is uncertain whether the differences between the expertise-based RCT results and the adjusted conventional RCT results, which assume the researchers know all of the points specified above, are clinically important in terms of patient-important outcomes.

Regarding the second issue questioning the applicability of the results of an expertise-based RCT, we encourage consideration of the paper written by Dr. Devereaux and colleagues and published in the British Medical Journal [2]. Briefly, in that paper, the authors described the difference between explanatory and pragmatic approaches to clinical trials emphasizing the expertise-based trial can be explanatory (limited to only surgeons with advanced expertise in ideal clinical settings) or pragmatic (including surgeons with at least basic competence in routine clinical settings). The applicability of the results of an expertisebased trial would relate specifically to this feature of the design. Thus, researchers should clearly describe the criteria for defining expertise. This issue of applicability is the same for conventional trials.

Regarding Dr. Biau and Professor Porcher's final point about the feasibility of conducting expertise-based trials, we have knowledge of at least one national multicenter expertise-based trial investigating the effectiveness of an orthopaedic intervention in the shoulder. At one center, patients referred to any of the participating surgeons are seen in a clinic that is run by the fellows to determine eligibility, and if eligible, to describe the study including the study design. In another center, a similar sort of setup is run by a primary care physician with expertise in sports medicine. Approximately 5\% of patients screened have 
refused for the reasons suggested by Drs. Biau and Porcher. It is unknown how many of these patients would have proved eligible for the trial; we can only report that approximately $50 \%$ of patients are found ineligible at the time of surgery (usually because of concomitant disease that precludes participation). Thus, the magnitude of the loss of potential participants may be much less. Time will tell whether the experience of researchers participating in other trials is similar to our experience.

\section{References}

1. Bednarska E, Bryant D, Devereaux, PJ. Orthopaedic surgeons prefer to participate in expertise-based randomized trials. Clin Orthop Relat Res. 2008;466:1734-1744.

2. Devereaux PJ, Bhandari M, Clarke M, Montori VM, Cook DJ, Yusuf S, Sackett DL, Cinà CS, Walter SD, Haynes B, Schünemann HJ, Norman GR, Guyatt GH. Need for expertise based randomised controlled trials. BMJ. 2005;330:88. 